\title{
Some sharp inequalities for multilinear integral operators
}

Daqing Lu*

"Correspondence:
Idqcslgdx@163.com
College of Mathematics, Changsha
University of Science and
Technology, Changsha, 410077, P.R.
China

\begin{abstract}
In this paper, some sharp inequalities for certain multilinear operators related to the Littlewood-Paley operator and the Marcinkiewicz operator are obtained. As an application, we obtain the $\left(L^{p}, L^{q}\right)$-norm inequalities and Morrey spaces boundedness for the multilinear operators.

MSC: 42B20; 42B25

Keywords: multilinear operator; Littlewood-Paley operator; Marcinkiewicz operator; Morrey space; $\mathrm{BMO}$
\end{abstract}

\section{Introduction and results}

In this paper, we study some multilinear operators related to some integral operators, whose definitions are as follows.

Fix $n>\delta \geq 0$. We denote $\Gamma(x)=\left\{(y, t) \in R_{+}^{n+1}:|x-y|<t\right\}$ and the characteristic function of $\Gamma(x)$ by $\chi_{\Gamma(x)}$. Suppose that $m_{j}$ are the positive integers $(j=1, \ldots, l), m_{1}+\cdots+m_{l}=m$ and $A_{j}$ are the functions on $R^{n}(j=1, \ldots, l)$. Let

$$
R_{m_{j}+1}\left(A_{j} ; x, y\right)=A_{j}(x)-\sum_{|\alpha| \leq m_{j}} \frac{1}{\alpha !} D^{\alpha} A_{j}(y)(x-y)^{\alpha} .
$$

Definition 1 Let $\varepsilon>0$ and $\psi$ be a fixed function which satisfies the following properties:

(1) $\int_{R^{n}} \psi(x) d x=0$,

(2) $|\psi(x)| \leq C(1+|x|)^{-(n+1-\delta)}$,

(3) $|\psi(x+y)-\psi(x)| \leq C|y|^{\varepsilon}(1+|x|)^{-(n+1+\varepsilon-\delta)}$ when $2|y|<|x|$.

The multilinear Littlewood-Paley operator is defined by

$$
S_{\psi}^{A}(f)(x)=\left[\iint_{\Gamma(x)}\left|F_{t}^{A}(f)(x, y)\right|^{2} \frac{d y d t}{t^{n+1}}\right]^{1 / 2},
$$

where

$$
F_{t}^{A}(f)(x, y)=\int_{R^{n}} \frac{\prod_{j=1}^{l} R_{m_{j}+1}\left(A_{j} ; x, z\right)}{|x-z|^{m}} \psi_{t}(y-z) f(z) d z
$$

(c) 2013 Lu; licensee Springer. This is an Open Access article distributed under the terms of the Creative Commons Attribution License (http://creativecommons.org/licenses/by/2.0), which permits unrestricted use, distribution, and reproduction in any medium, provided the original work is properly cited. 
and $\psi_{t}(x)=t^{-n+\delta} \psi(x / t)$ for $t>0$. Set $F_{t}(f)(y)=f * \psi_{t}(y)$. We also define that

$$
S_{\psi}(f)(x)=\left(\iint_{\Gamma(x)}\left|F_{t}(f)(y)\right|^{2} \frac{d y d t}{t^{n+1}}\right)^{1 / 2}
$$

which is the Littlewood-Paley operator (see [1]).

Let $H$ be the Hilbert space $H=\left\{h:\|h\|=\left(\iint_{R_{+}^{n+1}}|h(y, t)|^{2} d y d t / t^{n+1}\right)^{1 / 2}<\infty\right\}$. Then for each fixed $x \in R^{n}, F_{t}^{A}(f)(x, y)$ may be viewed as a mapping from $(0,+\infty)$ to $H$, and it is clear that

$$
S_{\psi}^{A}(f)(x)=\left\|\chi_{\Gamma(x)} F_{t}^{A}(f)(x, y)\right\|, \quad S_{\psi}(f)(x)=\left\|\chi_{\Gamma(x)} F_{t}(f)(y)\right\| .
$$

Definition 2 Let $0<\gamma \leq 1$ and $\Omega$ be homogeneous of degree zero on $R^{n}$ with $\int_{S^{n-1}} \Omega\left(x^{\prime}\right) d \sigma\left(x^{\prime}\right)=0$. Assume that $\Omega \in \operatorname{Lip}_{\gamma}\left(S^{n-1}\right)$, that is, there exists a constant $M>0$ such that for any $x, y \in S^{n-1},|\Omega(x)-\Omega(y)| \leq M|x-y|^{\gamma}$. The multilinear Marcinkiewicz operator is defined by

$$
\mu_{S}^{A}(f)(x)=\left[\iint_{\Gamma(x)}\left|F_{t}^{A}(f)(x, y)\right|^{2} \frac{d y d t}{t^{n+3}}\right]^{1 / 2},
$$

where

$$
F_{t}^{A}(f)(x, y)=\int_{|y-z| \leq t} \frac{\prod_{j=1}^{l} R_{m_{j}+1}\left(A_{j} ; x, z\right)}{|x-z|^{m}} \frac{\Omega(y-z)}{|y-z|^{n-1-\delta}} f(z) d z .
$$

Set

$$
F_{t}(f)(y)=\int_{|y-z| \leq t} \frac{\Omega(y-z)}{|y-z|^{n-1-\delta}} f(z) d z .
$$

We also define that

$$
\mu_{S}(f)(x)=\left(\iint_{\Gamma(x)}\left|F_{t}(f)(y)\right|^{2} \frac{d y d t}{t^{n+3}}\right)^{1 / 2}
$$

which is the Marcinkiewicz operator (see [2]).

Let $H$ be the Hilbert space $H=\left\{h:\|h\|=\left(\iint_{R_{+}^{n+1}}|h(y, t)|^{2} d y d t / t^{n+3}\right)^{1 / 2}<\infty\right\}$, then for each fixed $x \in R^{n}, F_{t}^{A}(f)(x, y)$ may be viewed as a mapping from $(0,+\infty)$ to $H$, and it is clear that

$$
\mu_{S}^{A}(f)(x)=\left\|\chi_{\Gamma(x)} F_{t}^{A}(f)(x, y)\right\|, \quad \mu_{S}(f)(x)=\left\|\chi_{\Gamma(x)} F_{t}(f)(y)\right\| .
$$

Note that when $m=0, S_{\psi}^{A}$ and $\mu_{S}^{A}$ are just the multilinear commutators (see [3, 4]). While when $m>0, S_{\psi}^{A}$ and $\mu_{S}^{A}$ are non-trivial generalizations of the commutators. It is well known that multilinear operators are of great interest in harmonic analysis and have been widely studied by many authors (see [5-9]). In [10], Hu and Yang proved a variant sharp estimate for the multilinear singular integral operators. In [11-13], authors proved a sharp estimate for the multilinear commutator. The main purpose of this paper is to prove the 
sharp inequalities for the multilinear integral operators $S_{\psi}^{A}$ and $\mu_{S}^{A}$ when $D^{\alpha} A_{j} \in B M O\left(R^{n}\right)$ for all $\alpha$ with $|\alpha|=m_{j}$. As an application, we obtain the $\left(L^{p}, L^{q}\right)$-norm inequalities and Morrey spaces boundedness for the multilinear operators.

First, let us introduce some notations. Throughout this paper, $Q$ will denote a cube of $R^{n}$ with sides parallel to the axes. For any locally integrable function $f$, the sharp function of $f$ is defined by

$$
f^{\#}(x)=\sup _{Q \ni x} \frac{1}{|Q|} \int_{Q}\left|f(y)-f_{Q}\right| d y,
$$

where, and in what follows, $f_{Q}=|Q|^{-1} \int_{Q} f(x) d x$. It is well-known that (see $\left.[14,15]\right)$

$$
f^{\#}(x)=\sup _{Q \ni x} \inf _{C \in C} \frac{1}{|Q|} \int_{Q}|f(y)-c| d y .
$$

We say that $f$ belongs to $B M O\left(R^{n}\right)$ if $f^{\#}$ belongs to $L^{\infty}\left(R^{n}\right)$ and $\|f\|_{B M O}=\left\|f^{\#}\right\|_{L^{\infty}}$. For $1 \leq$ $p<\infty$ and $0 \leq \delta<n$, let

$$
M_{\delta, p}(f)(x)=\sup _{Q \ni x}\left(\frac{1}{|Q|^{1-p \delta / n}} \int_{Q}|f(y)|^{p} d y\right)^{1 / p}
$$

we write that $M_{\mu}(f)=M_{n \mu, 1}(f)$, which is the fractional maximal operator.

Fixed $\lambda>0$. For $1 \leq p<\infty$, let

$$
\|f\|_{L^{p, \lambda}}=\sup _{x \in R^{n}, d>0}\left(\frac{1}{d^{\lambda}} \int_{B(x, d)}|f(y)|^{p} d y\right)^{1 / p}
$$

where $B(x, d)=\left\{y \in R^{n}:|x-y|<d\right\}$. The Morrey spaces are defined by (see [16-20])

$$
L^{p, \lambda}\left(R^{n}\right)=\left\{f \in L_{l o c}^{1}\left(R^{n}\right):\|f\|_{L^{p, \lambda}}<\infty\right\} .
$$

As the Morrey space may be considered as an extension of the Lebesgue space, it is natural and important to study the boundedness of the multilinear integral operator on the Morrey space.

We shall prove the following theorems.

Theorem 1 Let $D^{\alpha} A_{j} \in B M O\left(R^{n}\right)$ for all $\alpha$ with $|\alpha|=m_{j}$ and $j=1, \ldots, l$.

(1) Then there exists a constant $C>0$ such that for any $f \in C_{0}^{\infty}\left(R^{n}\right), 1<r<n / \delta$ and $x \in R^{n}$,

$$
\left(S_{\psi}^{A}(f)\right)^{\#}(x) \leq C \prod_{j=1}^{l}\left(\sum_{\left|\alpha_{j}\right|=m_{j}}\left\|D^{\alpha_{j}} A_{j}\right\|_{B M O}\right) M_{\delta, r}(f)(x) ;
$$

(2) If $1<p<n / \delta$ and $1 / p-1 / q=\delta / n$, then $S_{\psi}^{A}$ is bounded from $L^{p}\left(R^{n}\right)$ to $L^{q}\left(R^{n}\right)$, that is,

$$
\left\|S_{\psi}^{A}(f)\right\|_{L^{q}} \leq C \prod_{j=1}^{l}\left(\sum_{\left|\alpha_{j}\right|=m_{j}}\left\|D^{\alpha_{j}} A_{j}\right\|_{B M O}\right)\|f\|_{L^{p}} ;
$$


(3) If $1<p<n / \delta, 0<\lambda<n-p \delta, 1 / q=1 / p-\delta /(n-\lambda)$, then $S_{\psi}^{A}$ is bounded from $L^{p, \lambda}\left(R^{n}\right)$ to $L^{q, \lambda}\left(R^{n}\right)$, that is,

$$
\left\|S_{\psi}^{A}(f)\right\|_{L^{q, \lambda}} \leq C \prod_{j=1}^{l}\left(\sum_{\left|\alpha_{j}\right|=m_{j}}\left\|D^{\alpha_{j}} A_{j}\right\|_{B M O}\right)\|f\|_{L^{p, \lambda}} .
$$

Theorem 2 Let $D^{\alpha} A_{j} \in B M O\left(R^{n}\right)$ for all $\alpha$ with $|\alpha|=m_{j}$ and $j=1, \ldots, l$.

(1) Then there exists a constant $C>0$ such that for any $f \in C_{0}^{\infty}\left(R^{n}\right), 1<r<n / \delta$ and $x \in R^{n}$,

$$
\left(\mu_{S}^{A}(f)\right)^{\#}(x) \leq C \prod_{j=1}^{l}\left(\sum_{\left|\alpha_{j}\right|=m_{j}}\left\|D^{\alpha_{j}} A_{j}\right\|_{B M O}\right) M_{\delta, r}(f)(x) ;
$$

(2) If $1<p<n / \delta$ and $1 / p-1 / q=\delta / n$, then $\mu_{S}^{A}$ is bounded from $L^{p}\left(R^{n}\right)$ to $L^{q}\left(R^{n}\right)$, that is,

$$
\left\|\mu_{S}^{A}(f)\right\|_{L^{q}} \leq C \prod_{j=1}^{l}\left(\sum_{\left|\alpha_{j}\right|=m_{j}}\left\|D^{\alpha_{j}} A_{j}\right\|_{B M O}\right)\|f\|_{L^{p}}
$$

(3) If $1<p<n / \delta, 0<\lambda<n-p \delta, 1 / q=1 / p-\delta /(n-\lambda)$, then $\mu_{S}^{A}$ is bounded from $L^{p, \lambda}\left(R^{n}\right)$ to $L^{q, \lambda}\left(R^{n}\right)$, that is,

$$
\left\|\mu_{S}^{A}(f)\right\|_{L^{q, \lambda}} \leq C \prod_{j=1}^{l}\left(\sum_{\left|\alpha_{j}\right|=m_{j}}\left\|D^{\alpha_{j}} A_{j}\right\|_{B M O}\right)\|f\|_{L^{p, \lambda}} .
$$

Remark The conclusions of Theorems 1 and 2 are completely the same. Thus, they explain that the Littlewood-Paley and Marcinkiewicz operators have the many similar bondedness properties.

\section{Proofs of theorems}

To prove the theorems, we need the following lemmas.

Lemma 1 [7] Let $A$ be a function on $R^{n}$ and $D^{\alpha} A \in L^{q}\left(R^{n}\right)$ for all $\alpha$ with $|\alpha|=m$ and some $q>n$. Then

$$
\left|R_{m}(A ; x, y)\right| \leq C|x-y|^{m} \sum_{|\alpha|=m}\left(\frac{1}{|\tilde{Q}(x, y)|} \int_{\tilde{Q}(x, y)}\left|D^{\alpha} A(z)\right|^{q} d z\right)^{1 / q}
$$

where $\tilde{Q}$ is the cube centered at $x$ and having side length $5 \sqrt{n}|x-y|$.

Lemma 2 [21] Suppose that $1 \leq r<p<n / \delta$ and $1 / q=1 / p-\delta / n$. Then

$$
\left\|M_{\delta, r}(f)\right\|_{L^{q}} \leq C\|f\|_{L^{p}}
$$

Lemma 3 [16,17] Let $1<p<\infty$ and $0<\lambda<n$. Then the following estimates hold:

(a) $\|M(f)\|_{L^{p, \lambda}} \leq C\left\|f^{\#}\right\|_{L^{p, \lambda}}$;

(b) $\left\|M_{\mu}(f)\right\|_{L^{q, \lambda}} \leq C\|f\|_{L^{p, \lambda}}$ for $0<\mu<(n-\lambda) / n p$ and $1 / q=1 / p-n \eta /(n-\lambda)$. 
Lemma 4 Let $1<p<n / \delta$ and $1 / q=1 / p-\delta / n$. Then $S_{\psi}$ and $\mu_{S}$ are all bounded from $L^{p}\left(R^{n}\right)$ to $L^{q}\left(R^{n}\right)$.

Proof For $S_{\psi}$, by Minkowski inequality and the condition of $\psi$, we have

$$
\begin{aligned}
S_{\psi}(f)(x) & \leq \int_{R^{n}}|f(z)|\left(\int_{\Gamma(x)}\left|\psi_{t}(y-z)\right|^{2} \frac{d y d t}{t^{1+n}}\right)^{1 / 2} d z \\
& \leq C \int_{R^{n}}|f(z)|\left(\int_{0}^{\infty} \int_{|x-y| \leq t} \frac{t^{-2 n+2 \delta}}{(1+|y-z| / t)^{2 n+2-2 \delta}} \frac{d y d t}{t^{1+n}}\right)^{1 / 2} d z \\
& \leq C \int_{R^{n}}|f(z)|\left(\int_{0}^{\infty} \int_{|x-y| \leq t} \frac{2^{2 n+2} t^{1-n}}{(2 t+|y-z|)^{2 n+2-2 \delta}} d y d t\right)^{1 / 2} d z,
\end{aligned}
$$

noting that $2 t+|y-z| \geq 2 t+|x-z|-|x-y| \geq t+|x-z|$ when $|x-y| \leq t$ and

$$
\int_{0}^{\infty} \frac{t d t}{(t+|x-z|)^{2 n+2-2 \delta}}=C|x-z|^{-2 n+2 \delta}
$$

we obtain

$$
\begin{aligned}
S_{\psi}(f)(x) & \leq C \int_{R^{n}}|f(z)|\left(\int_{0}^{\infty} \frac{t d t}{(t+|x-z|)^{2 n+2-2 \delta}}\right)^{1 / 2} d z \\
& =C \int_{R^{n}} \frac{|f(z)|}{|x-z|^{n-\delta}} d z .
\end{aligned}
$$

For $\mu_{S}$, note that $|x-z| \leq 2 t,|y-z| \geq|x-z|-t \geq|x-z|-3 t$ when $|x-y| \leq t,|y-z| \leq t$, we have

$$
\begin{aligned}
\mu_{S}(f)(x) & \leq \int_{R^{n}}\left[\iint_{|x-y| \leq t}\left(\frac{|\Omega(y-z) \| f(z)|}{|y-z|^{n-1-\delta}}\right)^{2} \chi_{\Gamma(z)}(y, t) \frac{d y d t}{t^{n+3}}\right]^{1 / 2} d z \\
& \leq C \int_{R^{n}}|f(z)|\left[\iint_{|x-y| \leq t} \frac{\chi_{\Gamma(z)}(y, t) t^{-n-3}}{(|x-z|-3 t)^{2 n-2-2 \delta}} d y d t\right]^{1 / 2} d z \\
& \leq C \int_{R^{n}} \frac{|f(z)|}{|x-z|^{3 / 2}}\left[\int_{|x-z| / 2}^{\infty} \frac{d t}{(|x-z|-3 t)^{2 n-2}}\right]^{1 / 2} d z \\
& \leq C \int_{R^{n}} \frac{|f(z)|}{|x-z|^{n-\delta}} d z .
\end{aligned}
$$

Thus, the lemma follows from [21].

Proof of Theorem 1 (1) It suffices to prove for $f \in C_{0}^{\infty}\left(R^{n}\right)$ and some constant $C_{0}$, the following inequality holds:

$$
\frac{1}{|Q|} \int_{Q}\left|S_{\psi}^{A}(f)(x)-C_{0}\right| d x \leq C \prod_{j=1}^{l}\left(\sum_{\left|\alpha_{j}\right|=m_{j}}\left\|D^{\alpha_{j}} A_{j}\right\|_{B M O}\right) M_{\delta, r}(f)(x) .
$$

Without loss of generality, we may assume $l=2$. Fix a cube $Q=Q\left(x_{0}, d\right)$ and $\tilde{x} \in Q$. Let $\tilde{Q}=5 \sqrt{n} Q$ and $\tilde{A}_{j}(x)=A_{j}(x)-\sum_{|\alpha|=m_{j}} \frac{1}{\alpha !}\left(D^{\alpha} A_{j}\right) \tilde{Q}^{x^{\alpha}}$, then $R_{m_{j}}\left(A_{j} ; x, y\right)=R_{m_{j}}\left(\tilde{A}_{j} ; x, y\right)$ and 
$D^{\alpha} \tilde{A}_{j}=D^{\alpha} A_{j}-\left(D^{\alpha} A_{j}\right) \tilde{Q}$ for $|\alpha|=m_{j}$. We write, for $f_{1}=f \chi_{\tilde{Q}}$ and $f_{2}=f \chi_{R^{n}} \mid \tilde{Q}$,

$$
\begin{aligned}
F_{t}^{A}(f)(x, y)= & \int_{R^{n}} \frac{\prod_{j=1}^{2} R_{m_{j}+1}\left(\tilde{A}_{j} ; x, z\right)}{|x-z|^{m}} \psi_{t}(y-z) f(z) d z \\
= & \int_{R^{n}} \frac{\prod_{j=1}^{2} R_{m_{j}+1}\left(\tilde{A}_{j} ; x, z\right)}{|x-z|^{m}} \psi_{t}(y-z) f_{2}(z) d z \\
& +\int_{R^{n}} \frac{\prod_{j=1}^{2} R_{m_{j}}\left(\tilde{A}_{j} ; x, z\right)}{|x-z|^{m}} \psi_{t}(y-z) f_{1}(z) d z \\
& -\sum_{\left|\alpha_{1}\right|=m_{1}} \frac{1}{\alpha_{1} !} \int_{R^{n}} \frac{R_{m_{2}}\left(\tilde{A}_{2} ; x, z\right)(x-z)^{\alpha_{1}}}{|x-z|^{m}} D^{\alpha_{1}} \tilde{A}_{1}(z) \psi_{t}(y-z) f_{1}(z) d z \\
& -\sum_{\left|\alpha_{2}\right|=m_{2}} \frac{1}{\alpha_{2} !} \int_{R^{n}} \frac{R_{m_{1}}\left(\tilde{A}_{1} ; x, z\right)(x-z)^{\alpha_{2}}}{|x-z|^{m}} D^{\alpha_{2}} \tilde{A}_{2}(z) \psi_{t}(y-z) f_{1}(z) d z \\
& +\sum_{\left|\alpha_{1}\right|=m_{1},\left|\alpha_{2}\right|=m_{2}} \frac{1}{\alpha_{1} ! \alpha_{2} !} \int_{R^{n}} \frac{(x-z)^{\alpha_{1}+\alpha_{2}} D^{\alpha_{1}} \tilde{A}_{1}(z) D^{\alpha_{2}} \tilde{A}_{2}(z)}{|x-z|^{m}} \psi_{t}(y-z) f_{1}(z) d z,
\end{aligned}
$$

then

$$
\begin{aligned}
\left|S_{\psi}^{A}(f)(x)-S_{\psi}^{\tilde{A}}\left(f_{2}\right)\left(x_{0}\right)\right| & \\
= & \left|\left\|\chi_{\Gamma(x)} F_{t}^{A}(f)(x, y)\right\|-\left\|\chi_{\Gamma\left(x_{0}\right)} F_{t}^{\tilde{A}}\left(f_{2}\right)\left(x_{0}, y\right)\right\|\right| \\
\leq & \left\|\chi_{\Gamma(x)} F_{t}^{A}(f)(x, y)-\chi_{\Gamma\left(x_{0}\right)} F_{t}^{\tilde{A}}\left(f_{2}\right)\left(x_{0}, y\right)\right\| \\
\leq & \left\|\chi_{\Gamma(x)} \int_{R^{n}} \frac{\prod_{j=1}^{2} R_{m_{j}}\left(\tilde{A}_{j} ; x, z\right)}{|x-z|^{m}} \psi_{t}(y-z) f_{1}(z) d z\right\| \\
& +\left\|\chi_{\Gamma(x)} \sum_{\left|\alpha_{1}\right|=m_{1}} \frac{1}{\alpha_{1} !} \int_{R^{n}} \frac{R_{m_{2}}\left(\tilde{A}_{2} ; x, z\right)(x-z)^{\alpha_{1}}}{|x-z|^{m}} D^{\alpha_{1}} \tilde{A}_{1}(z) \psi_{t}(y-z) f_{1}(z) d z\right\| \\
& +\left\|\chi_{\Gamma(x)} \sum_{\left|\alpha_{2}\right|=m_{2}} \frac{1}{\alpha_{2} !} \int_{R^{n}} \frac{R_{m_{1}}\left(\tilde{A}_{1} ; x, z\right)(x-z)^{\alpha_{2}}}{|x-z|^{m}} D^{\alpha_{2}} \tilde{A}_{2}(z) \psi_{t}(y-z) f_{1}(z) d z\right\| \\
& +\left\|\chi_{\Gamma(x)} \sum_{\left|\alpha_{1}\right|=m_{1},\left|\alpha_{2}\right|=m_{2}} \frac{1}{\alpha_{1} ! \alpha_{2} !} \int_{R^{n}} \frac{(x-z)^{\alpha_{1}+\alpha_{2}} D^{\alpha_{1}} \tilde{A}_{1}(z) D^{\alpha_{2}} \tilde{A}_{2}(z)}{|x-z|^{m}} \psi_{t}(y-z) f_{1}(z) d z\right\| \\
& +\left\|\chi_{\Gamma(x)} F_{t}^{\tilde{A}}\left(f_{2}\right)(x, y)-\chi_{\Gamma\left(x_{0}\right)} F_{t}^{\tilde{A}}\left(f_{2}\right)\left(x_{0}, y\right)\right\| \\
:= & I_{1}(x)+I_{2}(x)+I_{3}(x)+I_{4}(x)+I_{5}(x),
\end{aligned}
$$

thus,

$$
\begin{aligned}
\frac{1}{|Q|} \int_{Q}\left|S_{\psi}^{A}(f)(x)-S_{\psi}^{\tilde{A}}\left(f_{2}\right)\left(x_{0}\right)\right| d x \\
\leq \frac{1}{|Q|} \int_{Q} I_{1}(x) d x+\frac{C}{|Q|} \int_{Q} I_{2}(x) d x+\frac{C}{|Q|} \int_{Q} I_{3}(x) d x \\
\quad+\frac{C}{|Q|} \int_{Q} I_{4}(x) d x+\frac{1}{|Q|} \int_{Q} I_{5}(x) d x \\
:=I_{1}+I_{2}+I_{3}+I_{4}+I_{5} .
\end{aligned}
$$


Now, let us estimate $I_{1}, I_{2}, I_{3}, I_{4}$ and $I_{5}$, respectively. First, for $x \in Q$ and $z \in \tilde{Q}$, by Lemma 1 , we get

$$
R_{m_{j}}\left(\tilde{A}_{j} ; x, z\right) \leq C|x-y|^{m_{j}} \sum_{\left|\alpha_{j}\right|=m_{j}}\left\|D^{\alpha_{j}} A_{j}\right\|_{B M O}
$$

Thus, by the $\left(L^{r}, L^{q}\right)$-boundedness of $S_{\psi}$, for $1<r<n / \delta$ and $1 / q=1 / r-\delta / n$, we obtain

$$
\begin{aligned}
I_{1} & \leq C \prod_{j=1}^{2}\left(\sum_{\left|\alpha_{j}\right|=m_{j}}\left\|D^{\alpha_{j}} A_{j}\right\|_{B M O}\right) \frac{1}{|Q|} \int_{Q}\left|S_{\psi}\left(f_{1}\right)(x)\right| d x \\
& \leq C \prod_{j=1}^{2}\left(\sum_{\left|\alpha_{j}\right|=m_{j}}\left\|D^{\alpha_{j}} A_{j}\right\|_{B M O}\right)\left(\frac{1}{|Q|} \int_{Q}\left|S_{\psi}\left(f_{1}\right)(x)\right|^{q} d x\right)^{1 / q} \\
& \leq C \prod_{j=1}^{2}\left(\sum_{\left|\alpha_{j}\right|=m_{j}}\left\|D^{\alpha_{j}} A_{j}\right\|_{B M O}\right)|Q|^{-1 / q}\left(\int_{Q}\left|f_{1}(x)\right|^{r} d x\right)^{1 / r} \\
& \leq C \prod_{j=1}^{2}\left(\sum_{\left|\alpha_{j}\right|=m_{j}}\left\|D^{\alpha_{j}} A_{j}\right\|_{B M O}\right) M_{\delta, r}(f)(\tilde{x}) .
\end{aligned}
$$

For $I_{2}$, denoting $r=p q$ for $1<p<n / \delta, q>1,1 / q+1 / q^{\prime}=1$ and $1 / s=1 / p-\delta / n$, we have, by Hölder's inequality,

$$
\begin{aligned}
I_{2} \leq & C \sum_{\left|\alpha_{2}\right|=m_{2}}\left\|D^{\alpha_{2}} A_{2}\right\|_{B M O} \sum_{\left|\alpha_{1}\right|=m_{1}} \frac{1}{|Q|} \int_{Q}\left|S_{\psi}\left(D^{\alpha_{1}} \tilde{A}_{1} f_{1}\right)(x)\right| d x \\
\leq & C \sum_{\left|\alpha_{2}\right|=m_{2}}\left\|D^{\alpha_{2}} A_{2}\right\|_{B M O} \sum_{\left|\alpha_{1}\right|=m_{1}}\left(\frac{1}{|Q|} \int_{R^{n}}\left|S_{\psi}\left(D^{\alpha_{1}} \tilde{A}_{1} f_{1}\right)(x)\right|^{s} d x\right)^{1 / s} \\
\leq & C \sum_{\left|\alpha_{2}\right|=m_{2}}\left\|D^{\alpha_{2}} A_{2}\right\|_{B M O} \sum_{\left|\alpha_{1}\right|=m_{1}}|Q|^{-1 / s}\left(\int_{R^{n}}\left|D^{\alpha_{1}} \tilde{A}_{1}(x) f_{1}(x)\right|^{p} d x\right)^{1 / p} \\
\leq & C \sum_{\left|\alpha_{2}\right|=m_{2}}\left\|D^{\alpha_{2}} A_{2}\right\|_{B M O} \\
& \times \sum_{\left|\alpha_{1}\right|=m_{1}}\left(\frac{1}{|Q|} \int_{\tilde{Q}}\left|D^{\alpha_{1}} \tilde{A}_{1}(x)\right|^{p q^{\prime}} d x\right)^{1 / p q^{\prime}}\left(\frac{1}{|Q|^{1-r \delta / n}} \int_{\tilde{Q}}|f(x)|^{p q} d x\right)^{1 / p q} \\
\leq & C \prod_{j=1}^{2}\left(\sum_{|\alpha|=m_{j}}\left\|D^{\alpha} A_{j}\right\|_{B M O}\right) M_{\delta, r}(f)(\tilde{x}) .
\end{aligned}
$$

For $I_{3}$, similar to the proof of $I_{2}$, we get

$$
I_{3} \leq C \prod_{j=1}^{2}\left(\sum_{|\alpha|=m_{j}}\left\|D^{\alpha} A_{j}\right\|_{B M O}\right) M_{\delta, r}(f)(\tilde{x}) .
$$


Lu Journal of Inequalities and Applications 2013, 2013:445

Page 8 of 14

http://www.journalofinequalitiesandapplications.com/content/2013/1/445

Similarly, for $I_{4}$, denoting $r=p q_{3}$ for $1<p<n / \delta, q_{1}, q_{2}, q_{3}>1,1 / q_{1}+1 / q_{2}+1 / q_{3}=1$ and $1 / s=1 / p-\delta / n$, we obtain

$$
\begin{aligned}
I_{4} \leq & C \sum_{\left|\alpha_{1}\right|=m_{1},\left|\alpha_{2}\right|=m_{2}} \frac{1}{|Q|} \int_{Q}\left|S_{\psi}\left(D^{\alpha_{1}} \tilde{A}_{1} D^{\alpha_{2}} \tilde{A}_{2} f_{1}\right)(x)\right| d x \\
\leq & C \sum_{\left|\alpha_{1}\right|=m_{1},\left|\alpha_{2}\right|=m_{2}}\left(\frac{1}{|Q|} \int_{R^{n}}\left|S_{\psi}\left(D^{\alpha_{1}} \tilde{A}_{1} D^{\alpha_{2}} \tilde{A}_{2} f_{1}\right)(x)\right|^{s} d x\right)^{1 / s} \\
\leq & C \sum_{\left|\alpha_{1}\right|=m_{1},\left|\alpha_{2}\right|=m_{2}}|Q|^{-1 / s}\left(\int_{R^{n}}\left|D^{\alpha_{1}} \tilde{A}_{1}(x) D^{\alpha_{2}} \tilde{A}_{2}(x) f_{1}(x)\right|^{p} d x\right)^{1 / p} \\
\leq & C \sum_{\left|\alpha_{1}\right|=m_{1},\left|\alpha_{2}\right|=m_{2}}\left(\frac{1}{|Q|} \int_{\tilde{Q}}\left|D^{\alpha_{1}} \tilde{A}_{1}(x)\right|^{p q_{1}} d x\right)^{1 / p q_{1}}\left(\frac{1}{|Q|} \int_{\tilde{Q}}\left|D^{\alpha_{2}} \tilde{A}_{2}(x)\right|^{p q_{2}} d x\right)^{1 / p q_{2}} \\
& \times\left(\frac{1}{|Q|^{1-r \delta / n}} \int_{\tilde{Q}}|f(x)|^{p q_{3}} d x\right)^{1 / p q_{3}} \\
\leq & C \prod_{j=1}^{2}\left(\sum_{|\alpha|=m_{j}}\left\|D^{\alpha} A_{j}\right\|_{B M O}\right) M_{\delta, r}(f)(\tilde{x}) .
\end{aligned}
$$

For $I_{5}$, we write

$$
\begin{aligned}
& \chi_{\Gamma(x)} F_{t}^{\tilde{A}}\left(f_{2}\right)(x, y)-\chi_{\Gamma\left(x_{0}\right)} F_{t}^{\tilde{A}}\left(f_{2}\right)\left(x_{0}, y\right) \\
& =\int_{R^{n}}\left(\chi_{\Gamma(x)}-\chi_{\Gamma\left(x_{0}\right)}\right) \frac{\prod_{j=1}^{2} R_{m_{j}}\left(\tilde{A}_{j} ; x, z\right)}{|x-z|^{m}} \psi_{t}(y-z) f_{2}(z) d z \\
& +\chi_{\Gamma\left(x_{0}\right)} \int_{R^{n}}\left(\frac{1}{|x-z|^{m}}-\frac{1}{\left|x_{0}-z\right|^{m}}\right) \prod_{j=1}^{2} R_{m_{j}}\left(\tilde{A}_{j} ; x, z\right) \psi_{t}(y-z) f_{2}(z) d z \\
& +\chi_{\Gamma\left(x_{0}\right)} \int_{R^{n}}\left(R_{m_{1}}\left(\tilde{A}_{1} ; x, z\right)-R_{m_{1}}\left(\tilde{A}_{1} ; x_{0}, z\right)\right) \frac{R_{m_{2}}\left(\tilde{A}_{2} ; x, z\right)}{\left|x_{0}-z\right|^{m}} \psi_{t}(y-z) f_{2}(z) d z \\
& +\chi_{\Gamma\left(x_{0}\right)} \int_{R^{n}}\left(R_{m_{2}}\left(\tilde{A}_{2} ; x, z\right)-R_{m_{2}}\left(\tilde{A}_{2} ; x_{0}, z\right)\right) \frac{R_{m_{1}}\left(\tilde{A}_{1} ; x_{0}, z\right)}{\left|x_{0}-z\right|^{m}} \psi_{t}(y-z) f_{2}(z) d z \\
& -\sum_{\left|\alpha_{1}\right|=m_{1}} \frac{1}{\alpha_{1} !} \int_{R^{n}}\left[\frac{R_{m_{2}}\left(\tilde{A}_{2} ; x, z\right)(x-z)^{\alpha_{1}} \chi_{\Gamma(x)}}{|x-z|^{m}}-\frac{R_{m_{2}}\left(\tilde{A}_{2} ; x_{0}, z\right)\left(x_{0}-z\right)^{\alpha_{1}} \chi_{\Gamma\left(x_{0}\right)}}{\left|x_{0}-z\right|^{m}}\right] \\
& \times D^{\alpha_{1}} \tilde{A}_{1}(z) \psi_{t}(y-z) f_{2}(z) d z \\
& -\sum_{\left|\alpha_{2}\right|=m_{2}} \frac{1}{\alpha_{2} !} \int_{R^{n}}\left[\frac{R_{m_{1}}\left(\tilde{A}_{1} ; x, z\right)(x-z)^{\alpha_{2}} \chi_{\Gamma(x)}}{|x-z|^{m}}-\frac{R_{m_{1}}\left(\tilde{A}_{1} ; x_{0}, z\right)\left(x_{0}-z\right)^{\alpha_{2}} \chi_{\Gamma\left(x_{0}\right)}}{\left|x_{0}-z\right|^{m}}\right] \\
& \times D^{\alpha_{2}} \tilde{A}_{2}(z) \psi_{t}(y-z) f_{2}(z) d z \\
& +\sum_{\left|\alpha_{1}\right|=m_{1},\left|\alpha_{2}\right|=m_{2}} \frac{1}{\alpha_{1} ! \alpha_{2} !} \int_{R^{n}}\left[\frac{(x-z)^{\alpha_{1}+\alpha_{2}} \chi_{\Gamma(x)}}{|x-z|^{m}}-\frac{\left(x_{0}-z\right)^{\alpha_{1}+\alpha_{2}} \chi_{\Gamma\left(x_{0}\right)}}{\left|x_{0}-z\right|^{m}}\right] \\
& \times D^{\alpha_{1}} \tilde{A}_{1}(z) D^{\alpha_{2}} \tilde{A}_{2}(z) \psi_{t}(y-z) f_{2}(z) d z \\
& =I_{5}^{(1)}+I_{5}^{(2)}+I_{5}^{(3)}+I_{5}^{(4)}+I_{5}^{(5)}+I_{5}^{(6)}+I_{5}^{(7)} \text {. }
\end{aligned}
$$


By Lemma 1 and the following inequality (see [15])

$$
\left|b_{Q_{1}}-b_{Q_{2}}\right| \leq C \log \left(\left|Q_{2}\right| /\left|Q_{1}\right|\right)\|b\|_{B M O} \quad \text { for } Q_{1} \subset Q_{2} \text {, }
$$

we know that, for $x \in Q$ and $z \in 2^{k+1} \tilde{Q} \backslash 2^{k} \tilde{Q}$,

$$
\begin{aligned}
\left|R_{m}(\tilde{A} ; x, z)\right| & \leq C|x-z|^{m} \sum_{|\alpha|=m}\left(\left\|D^{\alpha} A\right\|_{B M O}+\left|\left(D^{\alpha} A\right)_{\tilde{Q}(x, z)}-\left(D^{\alpha} A\right)_{\tilde{Q}}\right|\right) \\
& \leq C k|x-z|^{m} \sum_{|\alpha|=m}\left\|D^{\alpha} A\right\|_{B M O}
\end{aligned}
$$

Note that $|x-z| \sim\left|x_{0}-z\right|$ for $x \in Q$ and $z \in R^{n} \backslash \tilde{Q}$, we obtain, similar to the proof of Lemma 4,

$$
\begin{aligned}
& \left\|I_{5}^{(1)}\right\| \leq \int_{R^{n}}\left(\int \int _ { R _ { + } ^ { n + 1 } } \left[\frac{\prod_{j=1}^{2}\left|R_{m_{j}}\left(\tilde{A}_{j} ; x, z\right)\right|\left|\psi_{t}(y-z)\right|\left|f_{2}(z)\right|}{|x-z|^{m}}\right.\right. \\
& \left.\left.\times\left|\chi_{\Gamma(x)}(y, t)-\chi_{\Gamma\left(x_{0}\right)}(y, t)\right|\right]^{2} \frac{d y d t}{t^{n+1}}\right)^{1 / 2} d z \\
& \leq C \int_{R^{n}} \frac{\prod_{j=1}^{2}\left|R_{m_{j}}\left(\tilde{A}_{j} ; x, z\right)\right|\left|f_{2}(z)\right|}{\left|x_{0}-z\right|^{m}} \\
& \times\left|\int_{\Gamma(x)} \frac{t^{1-n} d y d t}{(t+|y-z|)^{2 n+2-2 \delta}}-\int_{\Gamma\left(x_{0}\right)} \frac{t^{1-n} d y d t}{(t+|y-z|)^{2 n+2-2 \delta}}\right|^{1 / 2} d z \\
& \leq C \int_{R^{n}} \frac{\prod_{j=1}^{2}\left|R_{m_{j}}\left(\tilde{A}_{j} ; x, z\right)\right|\left|f_{2}(z)\right|}{\left|x_{0}-z\right|^{m}} \\
& \times\left(\iint_{|y| \leq t}\left|\frac{1}{(t+|x+y-z|)^{2 n+2-2 \delta}}-\frac{1}{\left(t+\left|x_{0}+y-z\right|\right)^{2 n+2-2 \delta}}\right| \frac{d y d t}{t^{n-1}}\right)^{1 / 2} d z \\
& \leq C \int_{R^{n}} \frac{\prod_{j=1}^{2}\left|R_{m_{j}}\left(\tilde{A}_{j} ; x, z\right)\right|\left|f_{2}(z)\right|}{\left|x_{0}-z\right|^{m}}\left(\iint_{|y| \leq t} \frac{\left|x-x_{0}\right| t^{1-n} d y d t}{(t+|x+y-z|)^{2 n+3-2 \delta}}\right)^{1 / 2} d z \\
& \leq C \int_{R^{n}} \frac{\prod_{j=1}^{2} R_{m_{j}}\left(\tilde{A}_{j} ; x, z\right)\left|f_{2}(z)\right|\left|x-x_{0}\right|^{1 / 2}}{\left|x_{0}-z\right|^{m+n+1 / 2-\delta}} d z \\
& \leq C \prod_{j=1}^{2}\left(\sum_{|\alpha|=m_{j}}\left\|D^{\alpha} A_{j}\right\|_{B M O}\right) \sum_{k=0}^{\infty} \int_{2^{k+1} \tilde{Q} \backslash 2^{k} \tilde{Q}} k^{2} \frac{\left|x-x_{0}\right|^{1 / 2}}{\left|x_{0}-z\right|^{n+1 / 2-\delta}}|f(z)| d z \\
& \leq C \prod_{j=1}^{2}\left(\sum_{|\alpha|=m_{j}}\left\|D^{\alpha} A_{j}\right\|_{B M O}\right) \sum_{k=1}^{\infty} k^{2} 2^{-k / 2} \frac{1}{\left|2^{k} \tilde{Q}\right|^{1-\delta / n}} \int_{2^{k} \tilde{Q}}|f(z)| d z \\
& \leq C \prod_{j=1}^{2}\left(\sum_{|\alpha|=m_{j}}\left\|D^{\alpha} A_{j}\right\|_{B M O}\right) M_{\delta, r}(f)(\tilde{x}) \\
& \left\|I_{5}^{(2)}\right\| \leq C \int_{R^{n}} \frac{\left|x-x_{0}\right|}{\left|x_{0}-z\right|^{m+n+1-\delta}} \prod_{j=1}^{2}\left|R_{m_{j}}\left(\tilde{A}_{j} ; x, z\right)\right|\left|f_{2}(z)\right| d z \\
& \leq C \prod_{j=1}^{2}\left(\sum_{|\alpha|=m_{j}}\left\|D^{\alpha} A_{j}\right\|_{B M O}\right) \sum_{k=0}^{\infty} \int_{2^{k+1} \tilde{Q} \backslash 2^{k} \tilde{Q}} k^{2} \frac{\left|x-x_{0}\right|}{\left|x_{0}-z\right|^{n+1-\delta}}|f(z)| d z
\end{aligned}
$$


Lu Journal of Inequalities and Applications 2013, 2013:445

Page 10 of 14

http://www.journalofinequalitiesandapplications.com/content/2013/1/445

$$
\begin{aligned}
& \leq C \prod_{j=1}^{2}\left(\sum_{|\alpha|=m_{j}}\left\|D^{\alpha} A_{j}\right\|_{B M O}\right) \sum_{k=1}^{\infty} k^{2} 2^{-k} \frac{1}{\left|2^{k} \tilde{Q}\right|^{1-\delta / n}} \int_{2^{k} \tilde{Q}}|f(z)| d z \\
& \leq C \prod_{j=1}^{2}\left(\sum_{|\alpha|=m_{j}}\left\|D^{\alpha} A_{j}\right\|_{B M O}\right) M_{\delta, r}(f)(\tilde{x}) .
\end{aligned}
$$

For $I_{5}^{(3)}$ and $I_{5}^{(4)}$, by the formula (see [7])

$$
R_{m}(\tilde{A} ; x, z)-R_{m}\left(\tilde{A} ; x_{0}, z\right)=\sum_{|\beta|<m} \frac{1}{\beta !} R_{m-|\beta|}\left(D^{\beta} \tilde{A} ; x, x_{0}\right)(x-z)^{\beta}
$$

and Lemma 1, we have

$$
\left|R_{m}(\tilde{A} ; x, z)-R_{m}\left(\tilde{A} ; x_{0}, z\right)\right| \leq C \sum_{|\beta|<m} \sum_{|\alpha|=m}\left|x-x_{0}\right|^{m-|\beta|}|x-z|^{|\beta|}\left\|D^{\alpha} A\right\|_{B M O} .
$$

Thus, similar to the proof of Lemma 4,

$$
\begin{aligned}
\left\|I_{5}^{(3)}\right\| & \leq C \prod_{j=1}^{2}\left(\sum_{|\alpha|=m_{j}}\left\|D^{\alpha} A_{j}\right\|_{B M O}\right) \sum_{k=0}^{\infty} \int_{2^{k+1} \tilde{Q} \backslash 2^{k} \tilde{Q}} k \frac{\left|x-x_{0}\right|}{\left|x_{0}-z\right|^{n+1-\delta}}|f(y)| d y \\
& \leq C \prod_{j=1}^{2}\left(\sum_{|\alpha|=m_{j}}\left\|D^{\alpha} A_{j}\right\|_{B M O}\right) M_{\delta, r}(f)(\tilde{x}) ; \\
\left\|I_{5}^{(4)}\right\| & \leq C \prod_{j=1}^{2}\left(\sum_{|\alpha|=m_{j}}\left\|D^{\alpha} A_{j}\right\|_{B M O}\right) M_{\delta, r}(f)(\tilde{x}) .
\end{aligned}
$$

Similarly, we get

$$
\begin{aligned}
\left\|I_{5}^{(5)}\right\| \leq & C \sum_{\left|\alpha_{1}\right|=m_{1}} \int_{R^{n}} \|\left[\frac{R_{m_{2}}\left(\tilde{A}_{2} ; x, z\right)(x-z)^{\alpha_{1}} \chi_{\Gamma(x)}}{|x-z|^{m}}-\frac{\left.R_{m_{2}}\left(\tilde{A}_{2} ; x_{0}, z\right)\left(x_{0}-z\right)^{\alpha_{1}} \chi_{\Gamma\left(x_{0}\right)}\right]}{\left|x_{0}-z\right|^{m}}\right] \\
& \times \psi_{t}(y-z) \|\left|D^{\alpha_{1}} \tilde{A}_{1}(z)\right|\left|f_{2}(z)\right| d z \\
\leq & C \sum_{|\alpha|=m_{2}}\left\|D^{\alpha} A_{2}\right\|_{B M O} \sum_{\left|\alpha_{1}\right|=m_{1}} \sum_{k=1}^{\infty} k\left(2^{-k / 2}+2^{-k}\right) \\
& \times\left(\frac{1}{\left|2^{k} \tilde{Q}\right|} \int_{2^{k} \tilde{Q}}\left|D^{\alpha_{1}} \tilde{A}_{1}(y)\right|^{r^{\prime}} d y\right)^{1 / r^{\prime}}\left(\frac{1}{\left|2^{k} \tilde{Q}\right|^{1-r \delta / n}} \int_{2^{k} \tilde{Q}}|f(y)|^{r} d y\right)^{1 / r} \\
\leq & C \prod_{j=1}^{2}\left(\sum_{|\alpha|=m_{j}}\left\|D^{\alpha} A_{j}\right\|_{B M O}\right) M_{\delta, r}(f)(\tilde{x}) ; \\
\left\|I_{5}^{(6)}\right\| \leq & C \prod_{j=1}^{2}\left(\sum_{|\alpha|=m_{j}}\left\|D^{\alpha} A_{j}\right\|_{B M O}\right) M_{\delta, r}(f)(\tilde{x}) .
\end{aligned}
$$


For $I_{5}^{(7)}$, taking $q_{1}, q_{2}>1$ such that $1 / r+1 / q_{1}+1 / q_{2}=1$, then

$$
\begin{aligned}
\left\|I_{5}^{(7)}\right\| \leq & C \sum_{\left|\alpha_{1}\right|=m_{1},\left|\alpha_{2}\right|=m_{2}} \int_{R^{n}}\left\|\left[\frac{(x-z)^{\alpha_{1}+\alpha_{2}} \chi_{\Gamma(x)}}{|x-z|^{m}}-\frac{\left(x_{0}-z\right)^{\alpha_{1}+\alpha_{2}} \chi_{\Gamma\left(x_{0}\right)}}{\left|x_{0}-z\right|^{m}}\right] \psi_{t}(y-z)\right\| \\
& \times\left|D^{\alpha_{1}} \tilde{A}_{1}(z)\right|\left|D^{\alpha_{2}} \tilde{A}_{2}(z)\right|\left|f_{2}(z)\right| d z \\
\leq & C \sum_{\left|\alpha_{1}\right|=m_{1},\left|\alpha_{2}\right|=m_{2}} \sum_{k=1}^{\infty} k\left(2^{-k / 2}+2^{-k}\right)\left(\frac{1}{\left|2^{k} \tilde{Q}\right|^{1-p \delta / n}} \int_{2^{k} \tilde{Q}}|f(y)|^{r} d y\right)^{1 / r} \\
& \times\left(\frac{1}{\left|2^{k} \tilde{Q}\right|} \int_{2^{k} \tilde{Q}}\left|D^{\alpha_{1}} \tilde{A}_{1}(y)\right|^{q_{1}} d y\right)^{1 / q_{1}}\left(\frac{1}{\left|2^{k} \tilde{Q}\right|} \int_{2^{k} \tilde{Q}}\left|D^{\alpha_{2}} \tilde{A}_{2}(y)\right|^{q_{2}} d y\right)^{1 / q_{2}} \\
\leq & C \prod_{j=1}^{2}\left(\sum_{|\alpha|=m_{j}}\left\|D^{\alpha} A_{j}\right\|_{B M O}\right) M_{\delta, r}(f)(\tilde{x}) .
\end{aligned}
$$

Thus

$$
\left\|I_{5}\right\| \leq C \prod_{j=1}^{2}\left(\sum_{|\alpha|=m_{j}}\left\|D^{\alpha} A_{j}\right\|_{B M O}\right) M_{\delta, r}(f)(\tilde{x}) .
$$

We choose $1<r<p$ in (1), then (2) follows from Lemma 2. For (3), taking $1<r<\min (p,(n-$ $\lambda) / p \delta)$ in (1) and by Lemma 3 , we obtain

$$
\begin{aligned}
\left\|S_{\psi}^{A}(f)\right\|_{L^{q, \lambda}} & \leq C\left\|M\left(S_{\psi}^{A}(f)\right)\right\|_{L^{q, \lambda}} \leq C\left\|\left(S_{\psi}^{A}(f)\right)^{\#}\right\|_{L^{q, \lambda}} \\
& \leq C \prod_{j=1}^{2}\left(\sum_{|\alpha|=m_{j}}\left\|D^{\alpha} A_{j}\right\|_{B M O}\right)\left\|M_{\delta, r}(f)\right\|_{L^{q, \lambda}} \\
& \leq C \prod_{j=1}^{2}\left(\sum_{|\alpha|=m_{j}}\left\|D^{\alpha} A_{j}\right\|_{B M O}\right)\left\|\left(M_{r \delta / n}\left(|f|^{r}\right)\right)^{1 / r}\right\|_{L^{q, \lambda}} \\
& \leq C \prod_{j=1}^{2}\left(\sum_{|\alpha|=m_{j}}\left\|D^{\alpha} A_{j}\right\|_{B M O}\right)\left\|M_{r \delta / n}\left(|f|^{r}\right)\right\|_{L^{q / r, \lambda}}^{1 / r} \\
& \leq C \prod_{j=1}^{2}\left(\sum_{|\alpha|=m_{j}}\left\|D^{\alpha} A_{j}\right\|_{B M O}\right)\left\||f|^{r}\right\|_{L^{p / r, \lambda}}^{1 / r} \\
& \leq C \prod_{j=1}^{2}\left(\sum_{|\alpha|=m_{j}}\left\|D^{\alpha} A_{j}\right\|_{B M O}\right)\|f\|_{L^{p, \lambda}} .
\end{aligned}
$$

This completes the proof of Theorem 1 .

Proof of Theorem 2 It is only to prove (1). Let $Q, \tilde{Q}, \tilde{A}_{j}(x), f_{1}$ and $f_{2}$ be the same as the proof of Theorem 1. We write

$$
\begin{aligned}
& F_{t}^{A}(f)(x, y) \\
& \quad=\int_{R^{n}} \frac{\prod_{j=1}^{2} R_{m_{j+1}}\left(\tilde{A}_{j} ; x, z\right)}{|x-z|^{m}} \frac{\Omega(y-z)}{|y-z|^{n-1-\delta}} f_{2}(z) d z
\end{aligned}
$$


Lu Journal of Inequalities and Applications 2013, 2013:445

Page 12 of 14

http://www.journalofinequalitiesandapplications.com/content/2013/1/445

$$
\begin{aligned}
& +\int_{R^{n}} \frac{\prod_{j=1}^{2} R_{m_{j}}\left(\tilde{A}_{j} ; x, z\right)}{|x-z|^{m}} \frac{\Omega(y-z)}{|y-z|^{n-1-\delta}} f_{1}(z) d z \\
& -\sum_{\left|\alpha_{1}\right|=m_{1}} \frac{1}{\alpha_{1} !} \int_{R^{n}} \frac{R_{m_{2}}\left(\tilde{A}_{2} ; x, z\right)(x-z)^{\alpha_{1}} D^{\alpha_{1}} \tilde{A}_{1}(z)}{|x-z|^{m}} \frac{\Omega(y-z)}{|y-z|^{n-1-\delta}} f_{1}(z) d z \\
& -\sum_{\left|\alpha_{2}\right|=m_{2}} \frac{1}{\alpha_{2} !} \int_{R^{n}} \frac{R_{m_{1}}\left(\tilde{A}_{1} ; x, z\right)(x-z)^{\alpha_{2}} D^{\alpha_{2}} \tilde{A}_{2}(z)}{|x-z|^{m}} \frac{\Omega(y-z)}{|y-z|^{n-1-\delta}} f_{1}(z) d z \\
& +\sum_{\left|\alpha_{1}\right|=m_{1},\left|\alpha_{2}\right|=m_{2}} \frac{1}{\alpha_{1} ! \alpha_{2} !} \int_{R^{n}} \frac{(x-z)^{\alpha_{1}+\alpha_{2}} D^{\alpha_{1}} \tilde{A}_{1}(z) D^{\alpha_{2}} \tilde{A}_{2}(z)}{|x-z|^{m}} \frac{\Omega(y-z)}{|y-z|^{n-1-\delta}} f_{1}(z) d z,
\end{aligned}
$$

then

$$
\begin{aligned}
& \frac{1}{|Q|} \int_{Q}\left|\mu_{S}^{A}(f)(x)-\mu_{S}^{\tilde{A}}\left(f_{2}\right)\left(x_{0}\right)\right| d x \\
& \leq \frac{1}{|Q|} \int_{Q}\left\|\chi_{\Gamma(x)} F_{t}^{A}(f)(x, y)-\chi_{\Gamma\left(x_{0}\right)} F_{t}^{\tilde{A}}\left(f_{2}\right)\left(x_{0}, y\right)\right\| d x \\
& \leq \frac{1}{|Q|} \int_{Q}\left\|\chi_{\Gamma(x)} \int_{R^{n}} \frac{\prod_{j=1}^{2} R_{m_{j}}\left(\tilde{A}_{j} ; x, z\right)}{|x-z|^{m}} \frac{\Omega(y-z)}{|y-z|^{n-1-\delta}} f_{1}(z) d z\right\| d x \\
& +\frac{1}{|Q|} \int_{Q} \| \chi_{\Gamma(x)} \sum_{\left|\alpha_{1}\right|=m_{1}} \frac{1}{\alpha_{1} !} \\
& \times \int_{R^{n}} \frac{R_{m_{2}}\left(\tilde{A}_{2} ; x, z\right)(x-z)^{\alpha_{1}} D^{\alpha_{1}} \tilde{A}_{1}(z)}{|x-z|^{m}} \frac{\Omega(y-z)}{|y-z|^{n-1-\delta}} f_{1}(z) d z \| d x \\
& +\frac{1}{|Q|} \int_{Q} \| \chi_{\Gamma(x)} \sum_{\left|\alpha_{2}\right|=m_{2}} \frac{1}{\alpha_{2} !} \\
& \times \int_{R^{n}} \frac{R_{m_{1}}\left(\tilde{A}_{1} ; x, z\right)(x-z)^{\alpha_{2}} D^{\alpha_{2}} \tilde{A}_{2}(z)}{|x-z|^{m}} \frac{\Omega(y-z)}{|y-z|^{n-1-\delta}} f_{1}(z) d z \| d x \\
& +\frac{1}{|Q|} \int_{Q} \| \chi_{\Gamma(x)} \sum_{\left|\alpha_{1}\right|=m_{1},\left|\alpha_{2}\right|=m_{2}} \frac{1}{\alpha_{1} ! \alpha_{2} !} \\
& \times \int_{R^{n}} \frac{(x-z)^{\alpha_{1}+\alpha_{2}} D^{\alpha_{1}} \tilde{A}_{1}(z) D^{\alpha_{2}} \tilde{A}_{2}(z)}{|x-z|^{m}} \frac{\Omega(y-z)}{|y-z|^{n-1-\delta}} f_{1}(z) d z \| d x \\
& +\frac{1}{|Q|} \int_{Q}\left\|\chi_{\Gamma(x)} F_{t}^{\tilde{A}}\left(f_{2}\right)(x, y)-\chi_{\Gamma\left(x_{0}\right)} F_{t}^{\tilde{A}}\left(f_{2}\right)\left(x_{0}, y\right)\right\| d x \\
& :=J_{1}+J_{2}+J_{3}+J_{4}+J_{5} \text {. }
\end{aligned}
$$

Similar to the proof of Theorem 1, we get

$$
\begin{aligned}
J_{1} & \leq C \prod_{j=1}^{2}\left(\sum_{\left|\alpha_{j}\right|=m_{j}}\left\|D^{\alpha_{j}} A_{j}\right\|_{B M O}\right) \frac{1}{|Q|} \int_{Q}\left|\mu_{S}\left(f_{1}\right)(x)\right| d x \\
& \leq C \prod_{j=1}^{2}\left(\sum_{\left|\alpha_{j}\right|=m_{j}}\left\|D^{\alpha_{j}} A_{j}\right\|_{B M O}\right)\left(\frac{1}{|Q|} \int_{Q}\left|\mu_{S}\left(f_{1}\right)(x)\right|^{q} d x\right)^{1 / q} \\
& \leq C \prod_{j=1}^{2}\left(\sum_{\left|\alpha_{j}\right|=m_{j}}\left\|D^{\alpha_{j}} A_{j}\right\|_{B M O}\right) M_{\delta, r}(f)(\tilde{x})
\end{aligned}
$$




$$
\begin{aligned}
J_{2} & \leq C \sum_{\left|\alpha_{2}\right|=m_{2}}\left\|D^{\alpha_{2}} A_{2}\right\|_{B M O} \sum_{\left|\alpha_{1}\right|=m_{1}} \frac{1}{|Q|} \int_{Q}\left|\mu_{S}\left(D^{\alpha_{1}} \tilde{A}_{1} f_{1}\right)(x)\right| d x \\
& \leq C \sum_{\left|\alpha_{2}\right|=m_{2}}\left\|D^{\alpha_{2}} A_{2}\right\|_{B M O} \sum_{\left|\alpha_{1}\right|=m_{1}}\left(\frac{1}{|Q|} \int_{R^{n}}\left|\mu_{S}\left(D^{\alpha_{1}} \tilde{A}_{1} f_{1}\right)(x)\right|^{s} d x\right)^{1 / s} \\
& \leq C \prod_{j=1}^{2}\left(\sum_{|\alpha|=m_{j}}\left\|D^{\alpha} A_{j}\right\|_{B M O}\right) M_{\delta, r}(f)(\tilde{x}) ; \\
J_{3} & \leq C \prod_{j=1}^{2}\left(\sum_{|\alpha|=m_{j}}\left\|D^{\alpha} A_{j}\right\|_{B M O}\right) M_{\delta, r}(f)(\tilde{x}) ; \\
J_{4} & \leq C \sum_{\left|\alpha_{1}\right|=m_{1},\left|\alpha_{2}\right|=m_{2}} \frac{1}{|Q|} \int_{Q}\left|\mu_{S}\left(D^{\alpha_{1}} \tilde{A}_{1} D^{\alpha_{2}} \tilde{A}_{2} f_{1}\right)(x)\right| d x \\
& \leq C \sum_{\left|\alpha_{1}\right|=m_{1},\left|\alpha_{2}\right|=m_{2}}\left(\frac{1}{|Q|} \int_{R^{n}}\left|\mu_{S}\left(D^{\alpha_{1}} \tilde{A}_{1} D^{\alpha_{2}} \tilde{A}_{2} f_{1}\right)(x)\right|^{s} d x\right)^{1 / s} \\
& \leq C \prod_{j=1}^{2}\left(\sum_{|\alpha|=m_{j}}\left\|D^{\alpha} A_{j}\right\|_{B M O}\right) M_{\delta, p}(f)(\tilde{x}) .
\end{aligned}
$$

For $J_{5}$, we write

$$
\begin{aligned}
\chi_{\Gamma(x)} & F_{t}^{\tilde{A}}\left(f_{2}\right)(x, y)-\chi_{\Gamma\left(x_{0}\right)} F_{t}^{\tilde{A}}\left(f_{2}\right)\left(x_{0}, y\right) \\
= & \int_{R^{n}}\left(\chi_{\Gamma(x)}-\chi_{\Gamma\left(x_{0}\right)}\right) \frac{\prod_{j=1}^{2} R_{m_{j}}\left(\tilde{A}_{j} ; x, z\right)}{|x-z|^{m}} \frac{\Omega(y-z)}{|y-z|^{n-1-\delta}} f_{2}(z) d z \\
& +\chi_{\Gamma\left(x_{0}\right)} \int_{R^{n}}\left(\frac{1}{|x-z|^{m}}-\frac{1}{\left|x_{0}-z\right|^{m}}\right) \prod_{j=1}^{2} R_{m_{j}}\left(\tilde{A}_{j} ; x, z\right) \frac{\Omega(y-z)}{|y-z|^{n-1-\delta}} f_{2}(z) d z \\
& +\chi_{\Gamma\left(x_{0}\right)} \int_{R^{n}}\left(R_{m_{1}}\left(\tilde{A}_{1} ; x, z\right)-R_{m_{1}}\left(\tilde{A}_{1} ; x_{0}, z\right)\right) \frac{R_{m_{2}}\left(\tilde{A}_{2} ; x, z\right)}{\left|x_{0}-z\right|^{m}} \frac{\Omega(y-z)}{|y-z|^{n-1-\delta}} f_{2}(z) d z \\
& +\chi_{\Gamma\left(x_{0}\right)} \int_{R^{n}}\left(R_{m_{2}}\left(\tilde{A}_{2} ; x, z\right)-R_{m_{2}}\left(\tilde{A}_{2} ; x_{0}, z\right)\right) \frac{R_{m_{1}}\left(\tilde{A}_{1} ; x_{0}, z\right)}{\left|x_{0}-z\right|^{m}} \frac{\Omega(y-z)}{|y-z|^{n-1-\delta}} f_{2}(z) d z \\
& -\sum_{\left|\alpha_{1}\right|=m_{1}} \frac{1}{\alpha_{1} !} \int_{R^{n}}\left[\frac{R_{m_{2}}\left(\tilde{A}_{2} ; x, z\right)(x-z)^{\alpha_{1}} \chi_{\Gamma(x)}}{|x-z|^{m}}-\frac{R_{m_{2}}\left(\tilde{A}_{2} ; x_{0}, z\right)\left(x_{0}-z\right)^{\alpha_{1}} \chi_{\Gamma\left(x_{0}\right)}}{\left|x_{0}-z\right|^{m}}\right] \\
& \times \frac{\Omega(y-z)}{|y-z|^{n-1-\delta}} D^{\alpha_{1}} \tilde{A}_{1}(z) f_{2}(z) d z \\
& -\sum_{\left|\alpha_{2}\right|=m_{2}} \frac{1}{\alpha_{2} !} \int_{R^{n}}\left[\frac{R_{m_{1}}\left(\tilde{A}_{1} ; x, z\right)(x-z)^{\alpha_{2}} \chi_{\Gamma(x)}}{|x-z|^{m}}-\frac{\left.R_{m_{1}}\left(\tilde{A}_{1} ; x_{0}, z\right)\left(x_{0}-z\right)^{\alpha_{2}} \chi_{\Gamma\left(x_{0}\right)}\right]}{\left|x_{0}-z\right|^{m}}\right. \\
& \times \frac{\Omega(y-z)}{|y-z|^{n-1-\delta}} D^{\alpha_{2}} \tilde{A}_{2}(z) f_{2}(z) d z \\
& +\sum_{\left|\alpha_{1}\right|=m_{1},\left|\alpha_{2}\right|=m_{2}} \frac{1}{|y-z|^{n-1-\delta}} D^{\alpha_{1} ! \alpha_{2} !} \tilde{A}_{1}(z) D_{R^{n}}\left[\frac{(x-z)^{\alpha_{2}+\alpha_{2}} \chi_{\Gamma(x)} \tilde{A}_{2}(z) f_{2}(z) d z .}{|x-z|^{m}}-\frac{\left(x_{0}-z\right)^{\alpha_{1}+\alpha_{2}} \chi_{\Gamma\left(x_{0}\right)}}{\left|x_{0}-z\right|^{m}}\right] \\
& \Omega(y-z)
\end{aligned}
$$


Then, similar to the proof of Lemma 4 and Theorem 1, we get

$$
\left\|J_{5}\right\| \leq C \prod_{j=1}^{2}\left(\sum_{|\alpha|=m_{j}}\left\|D^{\alpha} A_{j}\right\|_{B M O}\right) M_{\delta, r}(f)(\tilde{x}) .
$$

The same argument as the proof of Theorem 1 will give the proof of (2) and (3), we omit the details and finish the proof.

\section{Competing interests}

The author declares that he has no competing interests.

Received: 21 September 2012 Accepted: 10 May 2013 Published: 4 October 2013

\section{References}

1. Torchinsky, A: Real Variable Methods in Harmonic Analysis. Pure and Applied Math., vol. 123. Academic Press, New York (1986)

2. Torchinsky, A, Wang, S: A note on the Marcinkiewicz integral. Colloq. Math. 60/61, 235-243 (1990)

3. Liu, LZ: Weighted weak type estimates for commutators of Littlewood-Paley operator. Jpn. J. Math. 29(1), 1-13 (2003)

4. Liu, LZ: The continuity of commutators on Triebel-Lizorkin spaces. Integral Equ. Oper. Theory 49, 65-75 (2004)

5. Cohen, J: A sharp estimate for a multilinear singular integral on $R^{n}$. Indiana Univ. Math. J. 30, 693-702 (1981)

6. Cohen, J, Gosselin, J: On multilinear singular integral operators on $R^{n}$. Stud. Math. 72, 199-223 (1982)

7. Cohen, J, Gosselin, J: A BMO estimate for multilinear singular integral operators. III. J. Math. 30, 445-465 (1986)

8. Coifman, R, Meyer, Y: Wavelets, Calderón-Zygmund and Multilinear Operators. Cambridge Studies in Advanced Math., vol. 48. Cambridge University Press, Cambridge (1997)

9. Ding, Y, Lu, SZ: Weighted boundedness for a class rough multilinear operators. Acta Math. Sin. 17, 517-526 (2001)

10. Hu, G, Yang, DC: A variant sharp estimate for multilinear singular integral operators. Stud. Math. 141, 25-42 (2000)

11. Perez, C: Endpoint estimate for commutators of singular integral operators. J. Funct. Anal. 128, 163-185 (1995)

12. Pérez, C, Pradolini, G: Sharp weighted endpoint estimates for commutators of singular integral operators. Mich. Math. J. 49, 23-37 (2001)

13. Pérez, C, Trujillo-Gonzalez, R: Sharp weighted estimates for multilinear commutators. J. Lond. Math. Soc. 65, 672-692 (2002)

14. Garcia-Cuerva, J, Rubio de Francia, JL: Weighted Norm Inequalities and Related Topics. North-Holland Math., vol. 116. North-Holland, Amsterdam (1985)

15. Stein, EM: Harmonic Analysis: Real Variable Methods, Orthogonality and Oscillatory Integrals. Princeton University Press, Princeton (1993)

16. Chiarenza, F, Frasca, M: Morrey spaces and Hardy-Littlewood maximal function. Rend. Mat. 7, 273-279 (1987)

17. Fan, DS, Lu, SZ, Yang, DC: Boundedness of operators in Morrey spaces on homogeneous spaces and its applications. Acta Math. Sin. 14, 625-634 (1998)

18. Di FaZio, G, Ragusa, MA: Commutators and Morrey spaces. Boll. Unione Mat. Ital. 7(5-A), 323-332 (1991)

19. Di Fazio, G, Ragusa, MA: Interior estimates in Morrey spaces for strong solutions to nondivergence form equations with discontinuous coefficients. J. Funct. Anal. 112, 241-256 (1993)

20. Mizuhara, T: Boundedness of some classical operators on generalized Morrey spaces. In: Harmonic Analysis, Proceedings of a Conference Held in Sendai, Japan, pp. 183-189 (1990)

21. Chanillo, S: A note on commutators. Indiana Univ. Math. J. 31, 7-16 (1982)

doi:10.1186/1029-242X-2013-445

Cite this article as: Lu: Some sharp inequalities for multilinear integral operators. Journal of Inequalities and Applications 2013 2013:445.

\section{Submit your manuscript to a SpringerOpen ${ }^{0}$ journal and benefit from:}

- Convenient online submission

- Rigorous peer review

- Immediate publication on acceptance

Open access: articles freely available online

- High visibility within the field

- Retaining the copyright to your article

Submit your next manuscript at $>$ springeropen.com 